\title{
Inflationary Magnetogenesis with Broken Local $U(1)$ Symmetry
}

\author{
Guillem Domènech, Chunshan Lin, and Misao Sasaki
}

Yukawa Institute for Theoretical Physics, Kyoto University

\begin{abstract}
We point out that a successful inflationary magnetogenesis could be realised if we break the local $U(1)$ gauge symmetry during inflation. The effective electric charge is fixed as a fundamental constant, which allows us to obtain an almost scale invariant magnetic spectrum avoiding both the strong coupling and back reaction problems. We examine the corrections to the primordial curvature perturbation due to these stochastic electromagnetic fields and find that, at both linear and non-linear orders, the contributions from the electromagnetic field are negligible compared to those created from vacuum fluctuations. Finally, the U(1) gauge symmetry is restored at the end of inflation.
\end{abstract}

Introduction Magnetic fields are present throughout the universe and play an important role in many astrophysical process such as galaxy dynamics, pulsars, white dwarfs, and even black holes. However, its origin is still not well understood. Interestingly, in 2010, it was found by blazar observations that magnetic fields are present even in inter-galactic void regions, with the coherence length of a few Mpc and the field strength larger than $10^{-15} G[1-4]$. The origin of such large scale cosmic magnetic fields, has been a challenging problem for years. It was suggested that inflation could be a prime candidate for the production of large scale magnetic fields, provided the conformal invariance of the $U(1)$ gauge theory was broken during inflation [11]. For example, this is easily the case in higher dimensions, where the $U(1)$ gauge field is not conformally invariant. Many models are proposed so far but none of them is convincing enough, see 12 14 for recent reviews.

On the other hand, stochastic primordial magnetic fields might also leave many imprints on $\mathrm{CMB}$, such as spectral distortions [5, 6], modifications of temperature and polarization spectra, Faraday rotation of CMB polarization [7 9], and so on. The most recent observational constraint from CMB is roughly $B_{\mathrm{Mpc}}<10^{-9} G$ by Planck [10].

One of the simplest, gauge invariant and well studied model is described by the Lagrangian [11, 15]

$$
\mathcal{L}_{E M}=-\frac{1}{4} f^{2}(\phi) F_{\mu \nu} F^{\mu \nu},
$$

where $F_{\mu \nu} \equiv \partial_{\mu} A_{\nu}-\partial_{\nu} A_{\mu}$. This model is equivalent to Bekenstein's variable charge theory [16]. Unfortunately, to produce large enough amplitude of magnetic field at large scale, this model suffers from either back reaction or strong coupling problem [18]. Furthermore, the primordial stochastic electric and magnetic fields behave as isocurvature perturbations, and hence they may also contribute to the CMB temperature anisotropies at both linear and nonlinear orders. Requiring the absence of the back reaction and strong coupling problems, as well as the absence of too large isocurvature perturbations from the eletromagnetic field, yields that inflation must happen at an extremely low energy scale [19 24].
For instance, one of the most stringent lower bounds was found in [23], in which only a very fine tuned scenario at an energy scale of inflation as low as $10^{-2} \mathrm{GeV}$ can explain the observed void magnetic field today. Basically it either rules out almost all models of inflation or rules out the inflationary origin of large scale magnetic fields.

Magnetogenesis with broken $U(1)$ symmetry In this letter, we point out that all of the above problems could be avoided if the local $U(1)$ gauge symmetry is broken during inflation. In contrast to Bekenstein's variable charge theory [16], we demote the electric charge as a fundamental coupling constant by considering the following modified QED Lagrangian during inflation,

$$
\begin{aligned}
\mathcal{L}= & -\frac{1}{4} f^{2}(\phi) F_{\mu \nu} F^{\mu \nu}-e_{0} f(\phi) A_{\mu} \bar{\psi} \gamma^{\mu} \psi \\
& +i \bar{\psi} \gamma^{\mu}\left(\partial_{\mu}+\Gamma_{\mu}\right) \psi-m \bar{\psi} \psi,
\end{aligned}
$$

where $e_{0}$ is the value of the electric charge today, $\psi$ is a charged fermion and $\Gamma_{\mu}$ is the spin connection. Thus, the standard local $U(1)$ gauge symmetry in QED is certainly broken. The residual symmetries are global symmetries

$$
\begin{aligned}
\psi & \rightarrow \psi e^{i \alpha}, \\
A_{\mu} & \rightarrow \lambda A_{\mu}, \\
f(\phi) & \rightarrow \lambda^{-1} f(\phi),
\end{aligned}
$$

as well as the on-shell local symmetry

$$
A_{\mu} \rightarrow A_{\mu}+\partial_{\mu} \sigma
$$

A possible origin for the effective action (2) is a braneworld scenario, in which charged matter fields live on the brane and the gauge field lives in the bulk, though the actual realization seems rather non-trivial.

We also mention that the above on-shell gauge symmetry resembles the case of the on-shell diffeomorphism invariance in Einstein gravity. In contrast to the off-shell gauge symmetry of standard QED, the action is invariant under the above gauge transformation only when the equation of motion is imposed. The equation of motion of the gauge field reads

$$
\nabla_{\mu}\left(f^{2} F^{\mu \nu}\right)-e_{0} f \bar{\psi} \gamma^{\nu} \psi=0 .
$$


Taking the divergence of the above equation of motion, by noting that $\nabla_{\mu} \nabla_{\nu}\left(f^{2} F^{\mu \nu}\right) \equiv 0$, we must have

$$
\nabla_{\mu}\left(e_{0} f \bar{\psi} \gamma^{\mu} \psi\right)=0
$$

Under the gauge transformation (4), the variation of the action reads

$$
\begin{aligned}
& \delta_{g} S=-\int \sqrt{-g} e_{0} f \partial_{\mu} \sigma \bar{\psi} \gamma^{\mu} \psi \\
& \quad=-\int \sqrt{-g}\left[\nabla_{\mu}\left(e_{0} f \sigma \bar{\psi} \gamma^{\mu} \psi\right)-\sigma \nabla_{\mu}\left(e_{0} f \bar{\psi} \gamma^{\mu} \psi\right)\right]
\end{aligned}
$$

where $\delta_{g}$ denotes the variation $\delta_{g} A_{\mu}=\partial_{\mu} \sigma$. The first term is just the total derivative, and the second term gives the generalised current conservation equation (6), and thus we have $\delta_{g} S=0$. Similar to the case of GR, where the gauge symmetry is also on-shell symmetry, the anomaly would appear at the quantum level and spoil the gauge symmetry (4). However, by imposing a more fundamental symmetry (such as SUSY), similar to what people did in supergravity, in principle all loop corrections can be cancelled out and thus the anomaly may be removed. But this is a topic beyond the scope of the present paper.

Another way to express our model is to do the scaling $A_{\mu} \rightarrow f^{-1} A_{\mu}$. Our action is reduced to

$$
\begin{aligned}
\mathcal{L}= & -\frac{1}{4} F_{\mu \nu} F^{\mu \nu}+i \bar{\psi} \gamma^{\mu} D_{\mu} \psi-m \bar{\psi} \psi \\
& -F_{\mu \nu} Q^{\mu} A^{\nu}-\frac{1}{2} Q_{\mu} Q^{\mu} A_{\nu} A^{\nu}+\frac{1}{2}\left(Q_{\mu} A^{\mu}\right)^{2},
\end{aligned}
$$

where $Q_{\mu} \equiv-\partial_{\mu}(\ln f)$ and $D_{\mu} \equiv \partial_{\mu}+i e_{0} A_{\mu}+\Gamma_{\mu}$. Our theory is the standard QED but the gauge symmetry is explicitly broken by the scalar field $\phi$. A related example was studied in [26] 27]. Note that our model is free from the strong coupling problem because we have fixed the electric charge as a fundamental constant, as clear from the above form of the Lagrangian.

The global symmetry between $A_{\mu}$ and $f(\phi)$ is also broken if we introduce a potential to the inflaton $\phi$ (which may arise from the SUSY breaking), to generate the well-behaved kinetic coupling function $f(\phi)$. It is known that we can obtain a scale-invariant magnetic spectrum if $f^{2} \sim a^{4}[17,18]$. However, in this case if we integrate over the whole momentum space, the energy density of the magnetic field would diverge logarithmically [25]. The divergence becomes even much worse if we take into account the slow-roll correction. Thus we relax the assumption on the coupling function as

$$
f^{2} \sim a^{4} \cdot e^{(4 \epsilon+\nu) N}
$$

where $\epsilon \equiv-\dot{H} / H^{2}$ is the slow-roll parameter during inflation, which is assumed to be constant for simplicity, $\nu$ is a new parameter and we require $\nu \ll 1$ to generate a nearly scale-invariant magnetic spectrum. $N$ is the number of $e$-folds counted backward from the end of inflation, thus $N=0$ at the end of inflation.

In the momentum space, the action of the gauge field reads

$$
S=\frac{1}{2} \sum_{s} \int d \tau \int d^{3} k f^{2}(\phi)\left[\left(A_{k}^{s}\right)^{\prime}\left(A_{-k}^{s}\right)^{\prime}-k^{2} A_{k}^{s} A_{-k}^{s}\right]
$$

where $s$ is the polarization index, $\tau$ is the conformal time, and the prime ( $\left.{ }^{\prime}\right)$ denotes the derivative w.r.t. the conformal time. The canonical conjugate momentum of the gauge field is defined by

$$
\pi_{k}=\frac{\delta S}{\delta A_{k}^{\prime}}=f^{2} A_{-k}^{\prime}
$$

where we have omitted the polarization index for notational simplicity. We quantize our system by imposing the following canonical commutation relation,

$$
\left[A_{k_{1}}, \pi_{k_{2}}\right]=i \delta\left(\mathbf{k}_{1}-\mathbf{k}_{2}\right)
$$

In terms of the creation and annihilation operators, the gauge field is expanded as

$$
A_{k}=u_{k} a_{k}+u_{k}^{*} a_{-k}^{\dagger}
$$

where, due to the commutation relation (12), the mode function $u_{k}$ is normalised as

$$
u_{k} u_{k}^{* \prime}-u_{k}^{*} u_{k}^{\prime}=\frac{i}{f^{2}}
$$

Taking the variation of the action w.r.t the gauge field, the equation of motion reads

$$
u_{k}^{\prime \prime}+\frac{2 f^{\prime}}{f} u_{k}^{\prime}+k^{2} u_{k}=0 .
$$

At the subhorizon limit $k \tau \rightarrow-\infty$, the spacetime is asymptotically Minkowskian, and the term $\frac{2 f^{\prime}}{f}$ is much smaller than $k$. Thus we may assume the standard Minkowski vacuum, and the mode function can be given by the WKB solution,

$$
u_{k}=\frac{1}{f \sqrt{2 k}} e^{-i k \tau}
$$

where the pre-factor $1 / f \sqrt{2 k}$ is fixed by the KleinGordon normalisation condition (14). Due to the nearly exponential expansion of the background, the physical scale eventually exceeds the Hubble horizon and since $f^{\prime} / f=O\left(a^{\prime} / a\right)$, we can neglect the $k^{2}$ term in the superhorizon limit $k \tau \rightarrow 0$. In this limit, the solution is

$$
u_{k}=c_{1}+c_{2} \int_{0}^{\tau} \frac{d \tau}{f^{2}}
$$


where $c_{1}$ represents the amplitude of the constant mode, and $c_{2}$ the decaying mode. Given the coupling function (9), it implies that the energy density of the electric field, which is dominated by the decaying mode, is much smaller than that of the magnetic field. Hereafter we ignore the decaying mode $c_{2}$ and hence the electric field. The $c_{1}$ may be computed by matching the subhorizon and superhorizon solutions at horizon crossing,

$$
c_{1} \simeq \frac{1}{f_{k} \sqrt{2 k}},
$$

where $f_{k}$ is the coupling function at horizon crossing time of the wavenumber $k$. Thus the power spectrum of the magnetic field can be calculated as

$$
P_{B}(k)=\frac{k^{5} f^{2}}{\pi^{2} a^{4}}\left|u_{k}\right|^{2}=\frac{k^{4}}{2 \pi^{2} a^{4}} \cdot \frac{f^{2}}{f_{k}^{2}} .
$$

At horizon crossing, we have $k=a_{*} H_{*}=a_{*} H_{f} e^{\epsilon N}$, where $H_{f}$ is the Hubble constant at the end of inflation. Setting

$$
f^{2}(N)=\exp [-4 N+(4 \epsilon+\nu) N] ; \quad N>0,
$$

and $f^{2}=1$ at and after the end of inflation, the power spectrum is found as

$$
P_{B}(k ; N)=\frac{H(N)^{4}}{2 \pi^{2}} e^{\nu\left(N-N_{k}\right)} .
$$

We assume that $\nu>0$ so that we can have a convergent energy density of the magnetic field. The energy density of the magnetic field is given by integrating over the whole momentum space (with UV cutoff at $\left.k=a H \equiv k_{*}(N)\right)$,

$$
\begin{aligned}
\rho_{B}(N) & =\frac{1}{2} \int_{0}^{k_{*}(N)} \frac{d k}{k} P_{B} \\
& =\frac{H^{4}(N)}{4 \pi^{2}} e^{\nu N} \int_{N}^{\infty} d N_{k} e^{-\nu N_{k}} \\
& =\frac{H^{4}(N)}{4 \pi^{2} \nu} .
\end{aligned}
$$

By requiring that the energy density of the magnetic field is much smaller than background energy density, we have a constraint on the parameter $\nu$,

$$
\frac{H_{f}^{2}}{M_{p}^{2}} \ll \nu \ll 1,
$$

where the upper bound is set to ensure a nearly scaleinvariant magnetic spectrum, and $H_{f}$ is the Hubble constant at the end of inflation. We see that this is actually a very week constraint.

The current magnetic strength could be estimated as follows. Assume that inflation happened at GUT scale, $H_{f} \sim 10^{-6} M_{p}$, we have

$$
B \sim H_{f}^{2} \nu^{-1 / 2} \sim \nu^{-1 / 2} 10^{-12} M_{p}^{2} \sim \nu^{-1 / 2} 10^{46} G
$$

at the end of inflation. Assuming that all the energy of the inflaton is transfered to radiation at once at the end of inflation, we have

$$
T_{\mathrm{CMB}}^{4} \sim M_{p}^{2} H_{f}^{2}\left(a_{f} / a_{0}\right)^{4},
$$

where $T_{\mathrm{CMB}}$ is the CMB temperature today, $a_{f}$ is the scale factor at the end of inflation, and $a_{0}$ is the scale factor today. The energy density of the magnetic field evolves also as radiation, $\rho_{B} \sim a^{-4}$, and its strength today reads

$$
B_{0} \sim B\left(\frac{a_{f}}{a_{0}}\right)^{2} \sim B \times \frac{T_{\mathrm{CMB}}^{2}}{M_{p} H} \sim \nu^{-1 / 2} 10^{-12} G,
$$

which is sufficiently large to explain the large scale magnetic field in the void. The most recent observational constraint from $\mathrm{CMB}$ is roughly $B_{\mathrm{Mpc}}<10^{-9} G$ by Planck [10], and it translates to the constraint,

$$
10^{-6}<\nu \ll 1 \text {. }
$$

Let's end this section with a remark. We assume that after inflation, the inflaton $\phi$ is trapped at the bottom of potential and we recover the standard $U(1)$ gauge theory. In the radiation dominant phase, the presence of the high conductivity would only dissipate the electric field, and keep the magnetic field frozen at super horizon scale.

The corrections to the primordial perturbations The primordial curvature perturbation also receives contributions from the electromagnetic field. The contributions to both linear and non-linear perturbations may be estimated by the $\delta N$ formalism [28 32]. The $\delta N$ formalism is a very powerful tool for the understanding of the perturbative physics on cosmological scales. It is essentially equivalent to focusing on the leading order terms in spatial gradient expansion, called the separate universe approach. According to it, the evolution of the local Hubble patch is well-approximated by the evolution of an unperturbed universe. The curvature perturbation at $t=t_{f}$ is given by the perturbation in the number of $e$-folds between the initial flat slice at $t=t_{i}$ and a final comoving slice at $t=t_{f}$ when the universe is in the adiabatic limit,

$$
\mathcal{R}_{c}\left(t_{f}, \mathbf{x}\right)=\delta N \equiv N\left(t_{i} \rightarrow t_{f}, \mathbf{x}\right)-N_{0}\left(t_{i} \rightarrow t_{f}\right),
$$

where $N_{0} \equiv \ln \left[a\left(t_{f}\right) / a\left(t_{i}\right)\right]$ is the unperturbed number of $e$-folds. In our case, the inflationary expansion history is parameterized by the value of the scalar field $\phi$,

$$
N(\phi)=\int_{\phi}^{\phi_{f}} \frac{H(\tilde{\phi})}{\dot{\tilde{\phi}}} d \tilde{\phi} \simeq \int_{\phi_{f}}^{\phi} \frac{\rho_{\text {inf }}(\tilde{\phi})+\rho_{B}(\tilde{\phi})}{M_{p}^{2} V_{\phi}(\tilde{\phi})} d \tilde{\phi},
$$

where $\rho_{\text {inf }}$ is the energy density of the inflaton, $V_{\phi} \equiv$ $\partial V / \partial \phi$, and we have used the Friedmann equation, the equation of motion for $\phi$, and the slow-roll approximation. According to the $\delta N$ formalism, we have

$$
\mathcal{R}_{c}\left(t_{f}, \mathbf{x}\right)=\frac{\partial N}{\partial \phi} \delta \phi=\frac{\rho_{\mathrm{inf}}(\phi) \delta \phi}{V_{\phi}(\phi)}+\frac{\rho_{B}(\phi) \delta \phi}{V_{\phi}(\phi)},
$$


where $\delta \phi$ is to be evaluated on the initial flat slice at $t=t_{i}$. To evaluate the curvature perturbation for a given $k$, we simply identify $t_{i}$ with the horizon crossing time determined by $a\left(t_{k}\right) H\left(t_{k}\right)=k$. The first term in (30) is due to the inflaton density fluctuation, and the second term is the additional contribution from the magnetic field. Given the constraint on the parameter $\nu$, (27), the contribution from the magnetic field is completely negligible. For instance, for parameter $\nu \sim \mathcal{O}\left(10^{-2}\right)$, we have $\rho_{B} / \rho_{\text {inf }} \sim 10^{-12}$.

The same conclusion applies to the non-linear perturbations. For the bi-spectrum (3-point function), the size of non-Gaussianity is characterised by the non-linear parameter

$$
-\frac{3}{5} f_{N L}=\frac{1}{2} \frac{\partial^{2} N / \partial \phi^{2}}{(\partial N / \partial \phi)^{2}} \supset \frac{3 H \dot{\rho}_{B}-\rho_{B} V^{\prime \prime}}{\rho_{\mathrm{inf}}^{2}}+\ldots
$$

Taking into account that the energy density of the magnetic field is much smaller than that of the inflaton field, and it evolves very slowly, the contribution to the nonGaussianity from the magnetic field is also completely negligible. The same conclusion can be trivially extended to the tri-spectrum (4-point function).

The magnetic field may also source tensor perturbations [23, 25]. The transverse and traceless part of the electromagnetic energy-momentum tensor appears as a source term on the right-hand side of the equation of motion for the tensor perturbation,

$$
\frac{1}{2 a^{2}}\left(\gamma_{i j}^{\prime \prime}+\frac{2 a^{\prime}}{a} \gamma_{i j}^{\prime}+k^{2} \gamma_{i j}\right) \simeq-\frac{1}{M_{p}^{2}}\left(B_{i} B_{j}\right)^{T T}
$$

As an order of magnitude estimation of the contribution from the electromagnetic field, we set the left-hand to be $H^{2} \gamma_{i j}$ and the right-hand side to be $\frac{H^{4}}{M_{p}^{2} \nu}$. Thus we obtain

$$
\gamma_{i j}^{\mathrm{em}} \sim \frac{H^{2}}{M_{p}^{2} \nu} \ll \gamma_{i j}^{\mathrm{vac}} \sim \frac{H}{M_{p}}
$$

where the middle double inequality holds because $\nu \gg$ $H / M_{p} \lesssim 10^{-6}$. Thus the vacuum fluctuation always dominates over the one generated by the electromagnetic field.

Conclusion and discussion The theoretical explanation on the origin of the large scale magnetic field has been a challenging problem for years. In this letter, we have pointed out that if we break the local $U(1)$ symmetry by promoting the effective electric charge to be a fundamental constant, the sufficient magnitude of stochastic magnetic fields may be generated during inflation without encountering neither the back reaction problem nor the strong coupling problem. The local $U(1)$ symmetry is restored at the end of inflation. We have examined that the contributions from the generated electromagnetic field to the primordial curvature perturbation are completely negligible both at linear and non-linear orders.

An additional interesting fact is that a broken $U(1)$ symmetry may have a more significant consequence such as baryon asymmetry. The broken $U(1)$ symmetry may lead to the lepton number violation. The lepton number violation translates to the baryon number violation through the weak interaction which meditated by sphaelerons 33]. Thus we may create the baryon asymmetry by creating the lepton number during inflation. We hope to come back to this issue in the future.

Acknowledgments This work was supported in part by MEXT KAKENHI Grant Number 15H05888. CL is supported by JSPS fellowship. We would like to thank H. Firouzjahi, A. E. Romano, T. Tanaka, A. Vikman, M. Werner for helpful discussions.

[1] A. Neronov, I. Vovk, Science 328:73-75,2010, [arxiv: 1006.3504].

[2] F. Tavecchio, G. Ghisellini, G. Bonnoli and L. Foschini, MNRAS (2011) 414 (4): 3566-3576, arXiv:1009.1048.

[3] F. Tavecchio, G. Ghisellini, L. Foschini, G. Bonnoli, G. Ghirlanda and P. Coppi, MNRAS (2010) 406 (1): L70L74, arXiv:1004.1329.

[4] A. M. Taylor, I. Vovk, A. Neronov, Astronomy \& Astrophysics, Volume 529, 2011, arXiv:1101.0932.

[5] D. Puy and P. Peter, Int. J. Mod. Phys. D 7, 489 (1998) astro-ph/9802329.

[6] K. Jedamzik, V. Katalinic and A. V. Olinto, Phys. Rev. Lett. 85, 700 (2000) astro-ph/9911100.

[7] A. Kosowsky, T. Kahniashvili, G. Lavrelashvili and B. Ratra, Phys. Rev. D 71, 043006 (2005) astro-ph/0409767.

[8] A. P.S. Yadav, L. Pogosian, T. Vachaspati, Phys. Rev. D 86 (2012) 123009, arXiv:1207.3356.

[9] C. Bonvin, C. Caprini and R. Durrer, Phys. Rev. D 88, 083515 (2013) arXiv:1308.3348 [astro-ph.CO]].

[10] P. A. R. Ade et al. [Planck Collaboration], arXiv:1502.01594 [astro-ph.CO].

[11] M. S. Turner and L. M. Widrow, Phys. Rev. D 37 (1988) 2743.

[12] A. Kandus, K. E. Kunze and C. G. Tsagas, Phys. Rept. 505, 1 (2011) arXiv:1007.3891 [astro-ph.CO]].

[13] R. Durrer and A. Neronov, Astron. Astrophys. Rev. 21, 62 (2013) arXiv:1303.7121 [astro-ph.CO]].

[14] K. Subramanian, arXiv:1504.02311 [astro-ph.CO].

[15] B. Ratra, Astrophys. J. 391, L1 (1992).

[16] J.D. Bekenstein. Phys. Rev. D25 (1982) 1527

[17] K. Bamba and M. Sasaki, JCAP 0702, 030 (2007)

[18] V. Demozzi, V. Mukhanov and H. Rubinstein, JCAP 0908, 025 (2009) arXiv:0907.1030 [astro-ph.CO]].

[19] T. Suyama and J. Yokoyama, Phys. Rev. D 86, 023512 (2012) arXiv:1204.3976 [astro-ph.CO]].

[20] T. Fujita and S. Mukohyama, JCAP 1210, 034 (2012) arXiv:1205.5031 [astro-ph.CO]].

[21] T. Fujita and S. Yokoyama, JCAP 1309, 009 (2013) arXiv:1306.2992 [astro-ph.CO]]. 
[22] T. Fujita and S. Yokoyama, JCAP 1403, 013 (2014) [JCAP 1405, E02 (2014)] arXiv:1402.0596 [astroph.CO]].

[23] R. J. Z. Ferreira, R. K. Jain and M. S. Sloth, JCAP 1406, 053 (2014) arXiv:1403.5516 [astro-ph.CO]].

[24] D. Green and T. Kobayashi, arXiv:1511.08793 [astroph.CO].

[25] N. Barnaby, R. Namba and M. Peloso, Phys. Rev. D 85, 123523 (2012) arXiv:1202.1469 [astro-ph.CO]].

[26] J. Liu, Y. Wang and S. Zhou, JCAP 1508, 033 (2015) arXiv:1502.05138 [hep-th]].

[27] G. Tasinato, JCAP 1503 (2015) 040 arXiv:1411.2803 [hep-th]].
[28] M. Sasaki and E. D. Stewart, Prog. Theor. Phys. 95, 71 (1996);

[29] A. A. Starobinsky, Phys. Lett. B 117, 175 (1982);

[30] A. A. Starobinsky, JETP Lett. 42, 152 (1985) [Pisma Zh. Eksp. Teor. Fiz. 42, 124 (1985)].

[31] D. H. Lyth, K. A. Malik, and M. Sasaki, JCAP 0505, 004 (2005).

[32] D. H. Lyth and Y. Rodriguez, Phys. Rev. Lett. 95, 121302 (2005) astro-ph/0504045.

[33] M. Fukugita and T. Yanagida, Phys. Lett. B 174, 45 (1986). 